\title{
Produção de fitomassa e teor de óleo essencial de folhas de alecrim-pimenta (Lippia sidoides Cham.) em diferentes espaçamentos de plantio
}

\author{
MELO, M.T.P.*; CARVALHO JÚNIOR, W.G.O.; SOUZA, M.F.; FIGUEIREDO, L.S.; MARTINS, E.R. \\ Universidade Federal de Minas Gerais, Instituto de Ciências Agrárias, Avenida Universitária, 1000, Bairro \\ Universitário, CEP:39400-000, Caixa Postal 135, Montes Claros-Brasil *mtuliomelo@hotmail.com
}

\begin{abstract}
RESUMO: O alecrim-pimenta, espécie nativa do nordeste brasileiro, é uma planta medicinal de porte arbustivo, com folhas aromáticas, que possuem óleo essencial rico em timol e carvacrol. Essas substâncias conferem grande importância à planta, uma vez que apresentam atividades farmacológicas comprovadas cientificamente. Desta forma, o estudo das condições de cultivo da espécie é essencial para obtenção de maiores produtividades. O espaçamento entre plantas destaca-se como um dos fatores mais importantes relativos ao cultivo, de forma que a produtividade e o rendimento da cultura são afetados pela densidade de plantas. Dada a necessidade de otimizar técnicas de cultivo do alecrim-pimenta (Lippia sidoides Cham.), objetivou-se avaliar a produção de fitomassa e óleo essencial em quatro espaçamentos de plantio. O trabalho foi realizado em campo, no Instituto de Ciências Agrárias da Universidade Federal de Minas Gerais (ICA/UFMG), no município de Montes Claros/MG. Utilizou-se o delineamento em blocos casualizados, com quatro tratamentos e cinco repetições. Os tratamentos foram definidos pelos espaçamentos de plantio: $1,0 \times 0,5 \mathrm{~m} ; 1,0 \times 1,0 \mathrm{~m} ; 1,5 \times 1,0 \mathrm{~m}$; e fileiras duplas $(1,0 \times 0,8 \times$ $0,5 \mathrm{~m}$ ), sendo $1,0 \mathrm{~m}$ entre fileiras duplas, $0,8 \mathrm{~m}$ entre fileiras simples e $0,5 \mathrm{~m}$ entre plantas na fileira. Após 150 dias do plantio no campo, foram avaliadas as variáveis produção de fitomassa fresca e seca das folhas, a produção e o teor de óleo essencial. Observou-se que o espaçamento de 1,0 x 0,5 m possibilitou uma maior produção de fitomassa e de óleo essencial, sendo, portanto, o mais indicado para o cultivo do alecrim-pimenta (Lippia sidoides Cham.).
\end{abstract}

Palavras-chave: Lippia sidoides, plantas medicinais, densidade de plantas

\begin{abstract}
Phytomass production and essential oil yield of pepper-rosmarin (Lippia sidoides Cham.) leaves in different planting spacings. Native to Northeastern Brazil, pepperrosmarin (Lippia sidoides Cham.) is a shrubby medicinal plant with aromatic leaves and essential oil rich in thymol and carvacrol. These components have great importance to the species since they present scientifically proven pharmacological activities. Thus, the study of cultivation conditions for this species is essential to obtain higher productivity. Spacing between plants is one of the most important factors related to cultivation, since crop productivity and yield are affected by plant density. Based on the need of optimizing pepper-rosmarin cultivation techniques, this work aimed to evaluate the phytomass production and the essential oil yield in four planting spacings. The experiment was carried out under field conditions in the Agricultural Sciences Institute, Federal University of Minas Gerais (ICA/UFMG), Montes Claros Municipality, Minas Gerais State, Brazil. Experimental design was in randomized blocks, with four treatments and five replicates. Treatments consisted of the planting spacings $1.0 \times 0.5 \mathrm{~m} ; 1.0 \times 1.0 \mathrm{~m} ; 1.5 \times 1.0 \mathrm{~m}$; and double rows $(1.0 \mathrm{x}$ $0.8 \times 0.5 \mathrm{~m}$ ), with $1.0 \mathrm{~m}$ between double rows, $0.8 \mathrm{~m}$ between single rows and $0.5 \mathrm{~m}$ between plants in the row. After 150 days of planting in the field, leaf fresh and dry phytomass production and essential oil yield were evaluated. The spacing $1.0 \times 0.5 \mathrm{~m}$ led to higher phytomass production and essential oil yield, being therefore the most recommended for pepper-rosmarin cultivation.
\end{abstract}

Key words: Lippia sidoides, medicinal plants, plant density

Recebido para publicação em 28/09/2009

Aceito para publicação em 05/07/2010

Rev. Bras. PI. Med., Botucatu, v.13, n.2, p.230-234, 2011. 


\section{INTRODUÇÃO}

O alecrim-pimenta (Lippia sidoides Cham.) é uma planta medicinal da família Verbenaceae, nativa do nordeste brasileiro, própria da vegetação do semiárido, que apresenta comportamento caducifólio, porte ereto, com até três metros de altura e possui folhas aromáticas e picantes, as quais contem óleo essencial rico em timol e carvacrol (Lorenzi \& Matos, 2008). Esses dois metabólitos apresentam atividade antibacteriana e antifúngica (Bruneton, 1999), conferindo à planta grande importância econômica. Considerada uma das sete espécies prioritárias da caatinga para conservação e manejo, a Lippia sidoides possui baixa exploração pelo homem (Vieira \& Silva, 2002). Entretanto, como a maioria das espécies nativas, ela é obtida por processos de extrativismo e é comercializada em barracas de raizeiros ou enviada a centros urbanos regionais (Scheffer et al., 1999).

As plantas competem diariamente por luz solar, água e nutrientes. Esses três fatores possuem papel fundamental no crescimento, desenvolvimento e produção da planta. Tal competição pode ser controlada pelas densidades de plantas por área, 0 que poderá implicar em diferentes produtividades (Taiz \& Zeiger, 2004).

Segundo Yadava (2001), o espaçamento entre plantas é um dos fatores mais importantes do cultivo, sendo que, a produtividade e o rendimento da cultura dependem do número de plantas por unidade de área.

Assim, o conhecimento prévio das condições de cultivo é de fundamental importância para estimular o aumento da produção dos componentes dos óleos essenciais (Lima et al., 2003) e, dessa forma, obter óleos essenciais com alta qualidade e, consequentemente, melhores valores de mercado.

Diversos estudos visando domesticar ou manejar o alecrim-pimenta vêm sendo conduzidos, incluindo-se adubação, propagação (Oliveira et al., 2008; Figueiredo et al., 2009; Carvalho Júnior et al., 2009a, b), curva de crescimento, sombreamento (Souza et al., 2007), etc. Assim, este estudo tem por objetivo avaliar a produção de fitomassa e de óleo essencial de alecrim-pimenta (L. sidoides) em quatro espaçamentos de plantio.

\section{MATERIAL E MÉTODO}

O presente trabalho foi realizado em campo, na Fazenda Experimental Hamilton de Abreu Navarro do Instituto de Ciências Agrárias da Universidade Federal de Minas Gerais, em Montes Claros, norte de Minas Gerais (1641'00"S e 4350'00'W).Na classificação de Koeppen (1948), o clima da região é o Aw, com uma estação seca bem definida e chuvas concentradas nos meses de novembro a janeiro.

O experimento foi conduzido sob o delineamento de blocos casualizados, com quatro tratamentos e cinco repetições, sendo 12 plantas úteis por parcela.

Os tratamentos foram definidos pelos seguintes espaçamentos de plantio: $1,0 \times 0,5 \mathrm{~m} ; 1,0$ $\times 1,0 \mathrm{~m} ; 1,5 \times 1,0 \mathrm{~m}$; e fileiras duplas $(1,0 \times 0,8 \times 0,5 \mathrm{~m})$, sendo $1,0 \mathrm{~m}$ entre fileiras duplas, $0,8 \mathrm{~m}$ entre fileiras simples e 0,5 m entre plantas na fileira (Figura 1 ).

As mudas foram obtidas através da propagação por estaquia, com a utilização de estacas apicais, com três nós, coletadas de plantas matrizes do Horto Medicinal do ICA/UFMG. As plantas foram

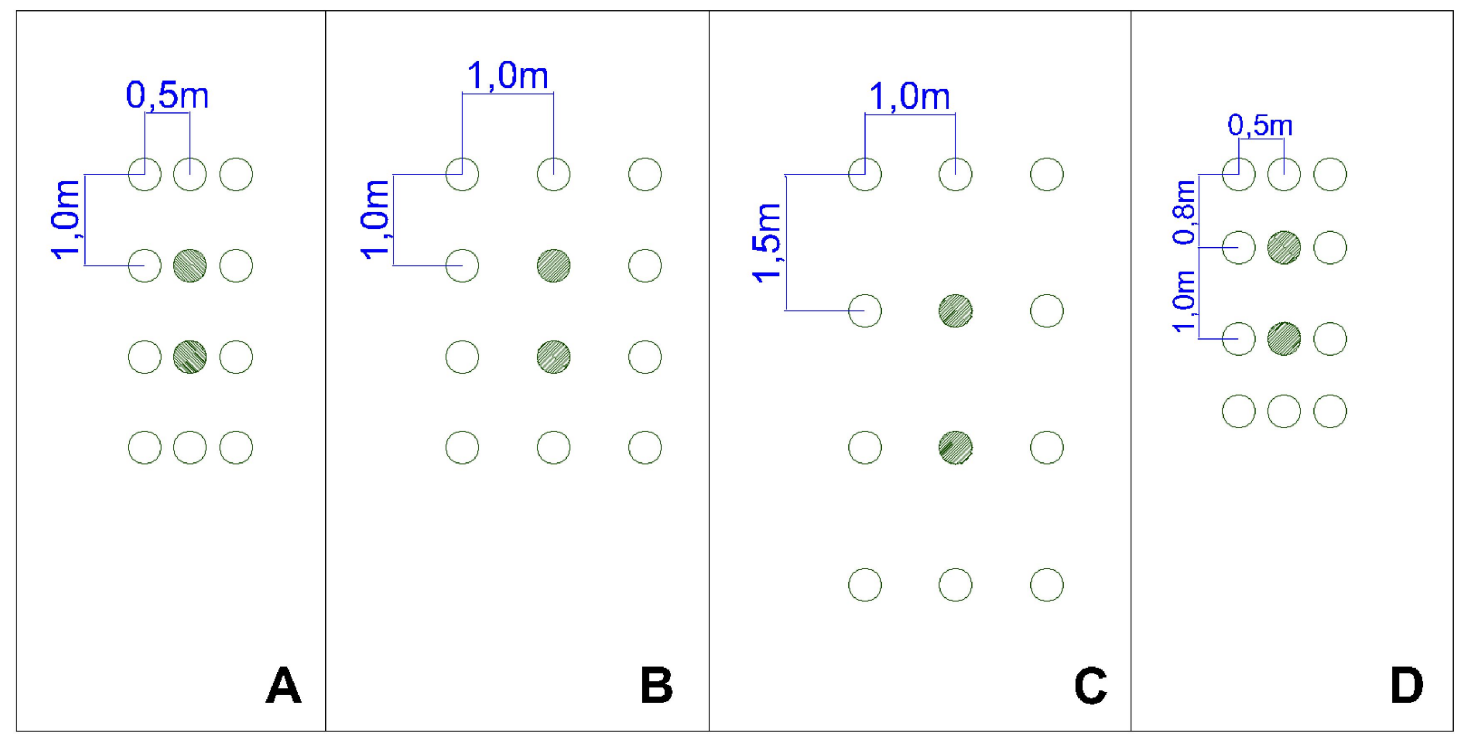

FIGURA 1. Croqui de um bloco do experimento com uma parcela de cada espaçamento: $1,0 \times 0,5 \mathrm{~m}(\mathrm{~A}) ; 1,0 \times 1,0$ $\mathrm{m}(\mathrm{B}) ; 1,5 \times 1,0 \mathrm{~m}(\mathrm{C})$; fileiras duplas (D). As circunferências hachuradas representam as plantas colhidas, enquanto as circunferências não hachuradas representam as bordaduras. 
produzidas em substrato comercial Plantmax®, em leito de enraizamento sob nebulização intermitente. Após 35 dias no leito de enraizamento, as mudas foram transferidas para copos plásticos, com capacidade de $200 \mathrm{~mL}$, preenchidos com solo da área de plantio e, após uma semana, foram transplantadas para covas de $30 \times 30 \times 30 \mathrm{~cm}$, devidamente adubadas com três quilos por metro quadrado $\left(3 \mathrm{~kg} \mathrm{~m}^{-2}\right)$ de esterco bovino curtido. O solo, que não recebeu correção de acidez, apresentava em pré-plantio, para a camada de 0 - $20 \mathrm{~cm}, 240 \mathrm{~g} \mathrm{~kg}^{-1}$ de argila, $15 \mathrm{~g} \mathrm{~kg}^{-1}$ de areia grossa e $525 \mathrm{~g} \mathrm{~kg}^{-1}$ de areia fina; $\mathrm{pH}=5,2 ; \mathrm{P}$ (Mehlich) = 0,9 $\mathrm{mg} \mathrm{kg}^{-1} ; \mathrm{K}=62 \mathrm{mg} \mathrm{kg}^{-1} ; \mathrm{Ca}, \mathrm{Mg} \mathrm{e} \mathrm{Al}$, 17,8 e $13 \mathrm{mmolc}^{-3}$, respectivamente; $V$ (saturação por bases) $=48 \% ; m$ (saturação por alumínio) $=33 \%$, e $25 \mathrm{~g} \mathrm{~kg}^{-1}$ de matéria orgânica.

Durante todo o período de cultivo do alecrimpimenta, foram realizadas irrigações por microaspersão, com duração de duas horas por dia, e controle de plantas espontâneas, por meio de capinas manuais.

A colheita foi realizada 150 dias após o transplantio das mudas, no período da manhã, com a coleta das duas plantas centrais de cada parcela, sendo o corte da planta toda, com a utilização de tesouras de poda. Em seguida, as amostras foram levadas ao Laboratório de Plantas Medicinais do ICA/ UFMG para avaliação da produção de matéria fresca e seca das folhas e da produção e teor de óleo essencial. Na determinação da matéria seca, as amostras foram mantidas em estufa de circulação forçada de ar a $65^{\circ} \mathrm{C}$, até massa constante.

O óleo essencial das folhas foi extraído pelo método de hidrodestilação, usando o aparelho de Clevenger, sendo utilizadas $60 \mathrm{~g}$ de folhas frescas por amostra, em balão de vidro de 1 litro, com cerca de $2 / 3$ do volume preenchido com água. O processo de extração teve a duração de $3 \mathrm{~h} \mathrm{e}$, em seguida, 0 óleo essencial foi separado da água com auxílio de micropipeta. Após esse processo, o material vegetal resultante foi mantido em estufa de circulação forçada de ar a $65^{\circ} \mathrm{C}$, até atingir peso constante. $\mathrm{O}$ teor de óleo essencial foi expresso com base na matéria seca da amostra utilizada na extração.

Os resultados do trabalho foram submetidos à análise de variância e, em seguida, as médias foram comparadas pelo teste Scott \& Knott, com a utilização do programa SAEG - Sistema para Análises Estatísticas e Genéticas (Ribeiro Júnior, 2001). Para a análise estatística, os dados de teor de óleo essencial foram previamente transformados para arco-seno $\sqrt{X / 100}$. Os dados foram apresentados como produtividade (produção por hectare), seguindo os tratamentos, de acordo com a seguinte formula: produtividade $\left(\mathrm{kg} \mathrm{ha}^{-1}\right)=$ [Produção total $(\mathrm{kg}) /$ densidade $\left.\left(\mathrm{m}^{2}\right)\right]^{*} 10.000 \mathrm{~m}^{2}$.

\section{RESULTADO E DISCUSSÃO}

A análise de variância (Tabela 1) apresentou diferenças significativas, pelo teste $F$, para as variáveis produção de óleo essencial (Óleo), fitomassa seca (FMS) e fresca (FMF) das folhas e teor de óleo essencial (Teor de óleo), em função dos quatro espaçamentos de plantio, sendo que a produção de óleo foi altamente significativa $(p<0,01)$.

Todos os espaçamentos avaliados não diferiram estatisticamente entre si em relação à variável teor de óleo, no entanto, o espaçamento de $1,0 \times 0,5 \mathrm{~m}$ destacou-se por apresentar as maiores médias para a maioria das características avaliadas (Tabela 2). Provavelmente, essa similaridade estatística está relacionada à proporcionalidade em relação ao aumento da produção de óleo e de fitomassa seca por área, ou seja, quanto maior a produção de fitomassa, maior a produção de óleo.

Em outros estudos com espaçamentos de plantio, envolvendo Cymbopogon flexuosus (Yadava, 2001) Cymbopogom citratus (Blank et al., 2007), também não se observaram diferenças significativas quanto ao teor de óleo essencial.

Em relação às produções de fitomassa fresca e fitomassa seca das folhas, pode-se observar que as maiores médias foram apresentadas pelo espaçamento $1,0 \times 0,5 \mathrm{~m}$, tratamento 1 (Tabela 2).

TABELA 1. Análise de variância para as variáveis produção de óleo essencial (Óleo), fitomassa seca (FMS), fitomassa fresca (FMF) e teor de óleo essencial (Teor de óleo) de alecrim-pimenta (Lippia sidoides Cham.) em função de quatro espaçamentos de plantio. ICA/UFMG, Montes Claros - MG. 20/07/2009.

\begin{tabular}{cccccc}
\hline \multirow{2}{*}{ Fontes de Variação } & \multirow{2}{*}{ G.L. } & \multicolumn{4}{c}{ Quadrados Médios } \\
\cline { 3 - 6 } & & Óleo & FMS & FMF & Teor de óleo \\
\cline { 3 - 6 } Blocos & 4 & 0,0083 & 598,02 & 3899,615 & 0,0008 \\
Espaçamento & 3 & $0,5846^{\star \star}$ & $3236,46^{\star}$ & $25656,78^{\star}$ & $0,005^{\star}$ \\
Resíduo & 11 & 0,0389 & 530,75 & 4671,512 & 0,0012 \\
C.V. (\%) & & 27,292 & 57,125 & 48,182 & 17,765 \\
\hline
\end{tabular}

${ }^{*}$ significativo a $5 \%$ de probabilidade pelo teste $\mathrm{F} .{ }^{* *}$ significativo a $1 \%$ de probabilidade pelo teste $\mathrm{F}$.

Rev. Bras. PI. Med., Botucatu, v.13, n.2, p.230-234, 2011. 
TABELA 2. Produção de óleo essencial (Óleo - $\mathrm{kg} \mathrm{ha}^{-1}$ ), fitomassa seca (FMS - $\mathrm{kg} \mathrm{ha}{ }^{-1}$ ), fitomassa fresca (FMF $\mathrm{kg} \mathrm{ha}^{-1}$ ) e teor de óleo essencial (\%) de alecrim-pimenta (Lippia sidoides Cham.) em função de quatro espaçamentos de plantio. ICA/UFMG, Montes Claros - MG. 20/07/2009

\begin{tabular}{cccccc}
\hline Espaçamento & $\begin{array}{c}\text { Densidade } \\
\left(\mathrm{m}^{2}\right)\end{array}$ & $\begin{array}{c}\text { Óleo } \\
\left(\mathrm{kg} \mathrm{ha}^{-1}\right)\end{array}$ & $\begin{array}{c}\mathrm{FMS} \\
\left(\mathrm{kg} \mathrm{ha}^{-1}\right)\end{array}$ & $\begin{array}{c}\text { FMF } \\
\left(\mathrm{kg} \mathrm{ha}^{-1}\right)\end{array}$ & $\begin{array}{c}\text { Teor de óleo } \\
(\%)\end{array}$ \\
\hline $1,0 \times 0,5 \mathrm{~m}$ & 0,5 & $20,89 \mathrm{a}$ & $841,91 \mathrm{a}$ & $2545,00 \mathrm{a}$ & $3,97 \mathrm{a}$ \\
$1,0 \times 1,0 \mathrm{~m}$ & 1,0 & $8,54 \mathrm{~b}$ & $316,65 \mathrm{~b}$ & $1278,00 \mathrm{~b}$ & $2,65 \mathrm{a}$ \\
$1,5 \times 1,0 \mathrm{~m}$ & 1,5 & $7,97 \mathrm{~b}$ & $177,19 \mathrm{~b}$ & $605,33 \mathrm{~b}$ & $5,50 \mathrm{a}$ \\
$1,0 \times 0,8 \times 0,5 \mathrm{~m}$ & 0,45 & $15,43 \mathrm{a}$ & $365,13 \mathrm{~b}$ & $1471,11 \mathrm{~b}$ & $3,78 \mathrm{a}$ \\
\hline
\end{tabular}

Médias seguidas pela mesma letra, nas colunas, não diferem estatisticamente entre si pelo teste Scott \& Knott, em nível de $5 \%$ de probabilidade.

Tal resultado é contrastante com os observados por Singh et al. (1996), ao trabalharem com Cymbopogon flexuosus, Vieira (2006), ao trabalhar com Cymbopogom citratus (DC) Stapf e Marco et al. (2006), ao trabalharem com Cymbopogon winterianus J., que observaram a maior produtividade no menor espaçamento de plantio, relacionando esse fato à competição das plantas por luz. Essa competição pode ter gerado plantas mais altas por unidade de área no menor espaçamento, com melhor interceptação da radiação solar, maior taxa de fotossíntese e menor quantidade de plantas espontâneas, consequentemente aumentando a eficiência da absorção de água e de nutrientes do solo (Taiz \& Zeiger, 2004).

No presente estudo, cada planta nos tratamentos referentes aos espaçamentos $1,0 \times 0,5$ $\mathrm{m}$ e fileiras duplas $(1,0 \times 0,8 \times 0,5)$ ocupava $0,5 \mathrm{~m}^{2} \mathrm{e}$ $0,45 \mathrm{~m}^{2}$, respectivamente, que são densidades de plantio muito aproximadas, o que, provavelmente foi causa da equivalência estatística, para a variável produção de óleo essencial, encontrada nesses tratamentos. Porém, a distribuição espacial do primeiro espaçamento $(1,0 \times 0,5 \mathrm{~m})$ apresenta maior uniformidade na captação da luz solar e, consequentemente, melhor absorção dessa radiação em relação ao segundo, formando plantas maiores e com maior produção de fitomassas fresca e seca.

O espaçamento de plantio apresentou expressiva influência sobre a produção de óleo essencial por hectare, sendo que os menores espaçamentos, $0,5 \times 1,0$ e fileiras duplas $(1,0 \times 0,5 \times$ $0,8)$, apresentaram produções estatisticamente superiores aos demais (Tabela 2). Resultado semelhante ao observado por Singh et al. (1996), que relacionaram o aumento do teor de óleo essencial ao incremento da fitomassa produzida nos menores espaçamentos. Como mencionado anteriormente, 0 fato de os tratamentos referentes aos menores espaçamentos terem apresentado médias estatisticamente equivalentes se deve à similaridade das densidades de plantio dessas.
Pode-se concluir que o espaçamento de plantio apresenta significativa influência sobre a produtividade de alecrim-pimenta (Lippia sidoides Cham.), sendo recomendado o espaçamento de 1,0 x $0,5 \mathrm{~m}$, de modo que se possa obter maiores produções de fitomassa e de óleo essencial.

\section{AGRADECIMENTO}

Os autores agradecem ao Conselho Nacional de Desenvolvimento Científico e Tecnológico (CNPq) pela concessão de bolsa a Melo, M.T.P. e ao Programa de Educação Tutorial (PET/Sesu/MEC) pela concessão de bolsa a Carvalho Júnior, W.G.O. Ao Fundo de Desenvolvimento Científico e Tecnológico do Banco do Nordeste do Brasil (BNBFUNDECI), pelo auxílio financeiro.

\section{REFERÊNCIA}

BLANK, A.F. et al. Densidades de plantio e doses de biofertilizante na produção de capim-limão. Horticultura Brasileira, v.25, n.3, p.343-9, 2007.

BRUNETON, J. Pharmacognosy, phytochemistry, medicinal plants, technique \& documentation. 2.ed. France: Lavoisier Publishing, 1999. 1119p.

CARVALHO JÚNIOR, W.G.O.; MELO, M.T.P.; MARTINS, E.R. Influência do horário de coleta de estacas e da nebulização intermitente na propagação vegetativa de alecrim-pimenta. Centro Científico Conhecer Enciclopédia Biosfera, v.5, n.8, p.1-4, 2009a.

CARVALHO JÚNIOR, W.G.O.; MELO, M.T.P.; MARTINS, E.R. Comprimento da estaca no desenvolvimento de mudas de alecrim-pimenta. Revista Ciência Rural, v.39, n.7, p.2199-202, 2009b.

FIGUEIREDO, L. S. et al. Influência do ácido indolbutírico no enraizamento de alecrim-pimenta (Lippia sidoides) em leito com umidade controlada. Revista Brasileira de Plantas Medicinais, v.11, n.1, p.33-6, 2009.

LIMA, H.R.P.; KAPLAN, M.A.R.; CRUZ, A.V.M. Influência dos fatores abióticos na produção e variabilidade de terpenóides em plantas. Revista Floresta e Ambiente, v.10, n.2, p.71-7, 2003. 
LORENZI, H.; MATOS, F.J.A. Plantas medicinais no Brasil: nativas e exóticas. Nova Odessa: Instituto Plantarum, 2008. 544p.

MARCO, C.A. et al. Influência de espaçamento, altura e época de corte no rendimento da biomassa e óleo essencial na cultura de capim citronela (Cymbopogon winterianus Jowitt.). Revista Ciência Agronômica, v.37, n.1, p.32-6, 2006.

OLIVEIRA, G.L. et al. Enraizamento de estacas de Lippia sidoides Cham. utilizando diferentes tipos de estacas, substratos e concentrações do ácido indolbutírico. Revista Brasileira de Plantas Medicinais, v.10, n.4, p.12-7, 2008. RIBEIRO JÚNIOR, J.I. Análises estatísticas no SAEG. Viçosa: UFV, 2001. 301p.

SCHEFFER, M.C.; MING, L.C.; ARAUJO, A.J. Conservação de recursos genéticos de plantas medicinais. In: QUEIROZ, M.A.; GOEDERT, C.O.; RAMOS, S.R.R. (Eds.). Recursos genéticos e melhoramento de plantas para o nordeste brasileiro. Petrolina-PE: Embrapa SemiÁrido, Brasília-DF: Embrapa Recursos Genéticos e Biotecnologia, 2009. Disponível em: <http://www.cpatsa. embrapa.br>. Acesso em: 24 abr. 2009.
SINGH, M.; SHIVARA, B.; SRIDHARA, S. Effect of plant spacing on Nitrogen levels on growth, herb, and yields of lemongrass (Cymbopogon flexuosus (Stend.) Wats. var. Cauvery). Journal of Agronomy and Crop Science, v.177, p.101-5, 1996.

SOUZA, M.F. et al. Influência do sombreamento na produção de fitomassa e óleo essencial em alecrimpimenta (Lippia sidoides Cham.). Revista Brasileira de Biociências, v.5, supl.2, p.108-10, 2007.

TAIZ, L.; ZEIGER, E. Fisiologia vegetal. Porto Alegre: Artmed, 2004. 720p.

VIEIRA, A.V. Influência do espaçamento, altura de corte e idade de primeiro corte na produtividade de capim santo. 2006. 37p. Dissertação (Mestrado em Agronomia/ Fitotecnia) - Universidade Federal do Ceará, Fortaleza. VIEIRA, R.F.; SILVA, S.R. Estratégias para conservação e manejo de recursos genéticos de plantas medicinais e aromáticas. Resultados da 1a Reunião Técnica. Brasília: Embrapa, 2002. 184p.

YADAVA, A.K. Cultivation of lemon grass (Cymbopogon flexuosus, 'CKP-25') under Poplar based agroforestry system. Indian Forester, v.127, p.213-23, 2001. 